\title{
$\mathrm{M}|\mathrm{R}| \mathrm{S}$ Internet Journal Nitride Semiconductor Research
}

\section{Room Temperature Ohmic contact on n-type GaN using plasma treatment}

\author{
Ho Won Jang ${ }^{1}$, Jong Kyu Kim ${ }^{1}$, Chang Min Jeon ${ }^{1}$ and Jong-Lam Lee ${ }^{1}$ \\ ${ }^{1}$ Pohang University of Science and Technology,
}

(Received Monday, January 10, 2000; accepted Monday, April 9, 2001)

Surface pretreatment using $\mathrm{Cl}_{2}$ plasma was applied to n-type $\mathrm{GaN}$ and Ti/Al ohmic contacts with resistivity of $\sim 10^{-6} \Omega \mathrm{cm}^{2}$, realized without annealing. Using synchrotron radiation photoemission spectroscopy, it was observed that the Fermi level moved by $0.5 \mathrm{eV}$ toward the conduction band edge and the atomic ratio of $\mathrm{Ga} / \mathrm{N}$ was increased by the treatment. This suggests that a number of $\mathrm{N}$ vacancies were produced at the treated surface and the Fermi level was pinned at the energy level of $\mathrm{N}$ vacancies near the conduction band. The $\mathrm{N}$ vacancies acting as donors for electrons produced a number of electrons, resulting in the near surface region to be in the degenerate state. Both the shift of Fermi level and the production of electrons at the treated surface lead to the reduction in contact resistivity through the decrease of the effective Schottky barrier for conduction of electrons.

\section{Introduction}

Ohmic contacts on GaN with a low contact resistivity attract continuously great interest in improving electrical and optical performance of various GaN-based devices such as optical devices and high temperature/ high power electric devices [1] [2] [3] [4] [5] [6] [7]. For n-type GaN, ohmic contacts with contact resistivity as low as $8 \times 10^{-6} \Omega \mathrm{cm}^{2}$ were achieved using Ti/Al metallization after annealing at $900{ }^{\circ} \mathrm{C}$ for $30 \mathrm{~s}$ [8]. Due to the formation of a TiN layer associated with the interaction of $\mathrm{Ti}$ with $\mathrm{GaN}$, a high concentration of $\mathrm{N}$ vacancies, $V_{N}$, could be created near the interface, causing the $\mathrm{GaN}$ to be heavily doped n-type. This Ti/Al metal scheme has been used as the standard ohmic contact on n-type GaN [9] [10].

In the fabrication of a device, high temperature annealing degrades the device performance. The development of nonalloyed contacts could prevent the device from such thermal degradation during the fabrication process. In addition, adopting the nonalloyed contact in the fabrication process of the device could lead to improvement in process freedom. The pretreatment of the surface prior to metal deposition plays a key role in reducing the contact resistivity on $\mathrm{GaN}$ [11]. A number of $\mathrm{Ga}$ vacancies, $\mathrm{V}_{\mathrm{Ga}}$, acting as an acceptor for electrons, were found at the surface region of p-type $\mathrm{GaN}$ when the surface of $\mathrm{GaN}$ were treated using aqua regia solution [12]. Thus, the Fermi level position shifts to an energy level of acceptor defects, resulting in a decease of contact resistivity from the decrease of the Schottky barrier height for hole transport. This suggests that room temperature ohmic contacts could be achieved through production of a donor-type defect, $\mathrm{V}_{\mathrm{N}}$ using proper surface treatment [13]. It has been shown that reactive ion etching $\mathrm{n}-\mathrm{GaN}$ surfaces improves the contact resistance [9] [13]. However, there is no reported work that would reveal the mechanism for ohmic contact formation.

In this work, we report a room temperature ohmic contact on n-type GaN with low contact resistivity using inductively coupled plasma (ICP) treatment. Synchrotron radiation photoemission spectroscopy (SRPES) was employed to examine the formation mechanism of the ohmic contact by plasma treatment on n-type $\mathrm{GaN}$.

\section{Experimental details}

GaN films used in this work were grown by metalorganic chemical vapor deposition on (0001) sapphire substrates. An undoped GaN buffer layer with a thickness of $50 \mathrm{~nm}$ was grown, followed by growth of $1-\mu \mathrm{m}-$ thick n-type GaN doped with $\mathrm{Si}$. The carrier concentration was determined to be $1 \times 10^{18} \mathrm{~cm}^{-3}$ by Hall measurements. Two types of samples were prepared. One set was prepared by deoxidizing the grown sample using $\mathrm{HCl}$ solution (HCl-treated) and the other was treated by ICP with $\mathrm{Cl}_{2}$ gas for $3 \mathrm{~min}$ (plasma-treated). In the plasma treatment, a base pressure of $5 \times 10^{-5}$ Torr and a 
flow rate of $10 \mathrm{sccm}$ were used. For the measurement by circular transmission line method (TLM), circular contact pads with a radius of $100 \mu \mathrm{m}$ were patterned on both samples using photoresist. This contact design avoids the need for isolation of the contact structures by implantation or etching [14]. The patterned samples were dipped into an $\mathrm{HCl}: \mathrm{H}_{2} \mathrm{O}(1: 1)$ solution for $3 \mathrm{~min}$, followed by deposition of $\mathrm{Ti}(300 \AA)$ and $\operatorname{Al}(1000 \AA)$ layers in sequence using an electron beam evaporator under a vacuum condition lower than $4 \times 10^{-7}$ Torr. A liftoff process was carried out to form a linear configuration of the TLM pads. Current-voltage (I-V) measurements were carried out by the four-point probe technique using a parameter analyzer (HP 4156A).

To investigate the chemical bonding state at the treated surface, SRPES was employed. Four types of samples were prepared for SRPES. One set was an asgrown sample, the second one was the plasma-treated sample, and the third and last set were prepared by dipping the as-grown and the plasma-treated samples into the $\mathrm{HCl}$ solution before loading to a UHV chamber. These schemes are consistent with the main steps in the preparation of both the HCl-treated and the plasmatreated samples for the electrical measurements. The change of stoichiometry at GaN surface after the plasma treatment was measured using angle resolved SRPES analysis.

\section{Results and Discussion}

\subsection{Electrical properties}

Resistances between pads for the HCl-treated and plasma-treated samples, measured at a voltage of $0 \mathrm{~V}$ between the ohmic pads, are summarized in Table I. The contact resistivity was determined from the linear plot of the measured resistance with gap spacing using the least-squares linear regression fitting method. The contact resistivity for the HCl-treated sample was as high as $\sim 10^{-1} \Omega \mathrm{cm}^{2}$, but it was decreased to $9.4 \times 10^{-6} \Omega \mathrm{cm}^{2}$ by the plasma treatment. To the best of our knowledge, the contact resistivity obtained at room temperature is the lowest reported and is comparable to previous results achieved through high temperature annealing [8].

\subsection{Surface analysis}

Figure 1(a) shows the deconvoluted Ga $3 d$ spectra with the type of surface treatment of n-type GaN, characterized using SRPES. The peak for the Ga-N bond shifted by $0.16 \mathrm{eV}$ toward higher binding energy after the $\mathrm{Cl}_{2}$ plasma treatment, while no peak shift was observed after the $\mathrm{HCl}$ treatment. The peak shift in $\mathrm{Ga}-\mathrm{O}$ bonds exhibits the same trend. The full-width-at-half-maximum (FWHM) of Ga-O bond is listed in Table II. The FWHM was decreased with the plasma treatment. This suggests that the chemical composition in Ga oxides on the plasma-treated surface should be different from that on the as-grown one. SRPES spectra of O $1 s$ are shown in Figure 1(b). The peak intensity corresponding to $\mathrm{O} 1 \mathrm{~s}$ photoelectrons was increased after the $\mathrm{Cl}_{2}$ plasma treatment. The area of $\mathrm{O} 1 \mathrm{~s}$ peak in the plasma-treated sample is larger by 1.37 times than that in the as-grown one. This suggests that $\mathrm{Ga}$ oxides were produced during the plasma treatment, mainly composed of $\mathrm{Ga}$ and $\mathrm{O}$ atoms. When the as-grown sample was treated with the $\mathrm{HCl}$ solution, the peak area of $\mathrm{O} 1 s$ was reduced by the factor of 0.88 . Only a small decrease $(1.5 \%)$ of the $\mathrm{O} 1 \mathrm{~s}$ peak in the $\mathrm{ICP}+\mathrm{HCl}$ treated sample was found. The results in Figs. 1(a) and (b) show that the Ga oxide was unintentionally formed during the plasma treatment and its chemical composition was changed, that is, the surface oxide produced during the plasma treatment might be composed of $\mathrm{Ga}-\mathrm{N}$ bonds as well as $\mathrm{Ga}-\mathrm{O}$ bonds. Figure 1(c) shows valence band spectra. The Fermi level was determined by linearly extrapolating the sloped region with the base line in the valence band spectrum of $\mathrm{Au}$ foil, which is defined as the level of zero binding energy. The Fermi level in the plasma-treated sample shifts toward conduction band by $0.5 \mathrm{eV}$ relative to that in the as-grown one, but there is no considerable shift after $\mathrm{HCl}$ treatments in both samples. The Fermi level shift implies the generation of surface states at the plasmatreated surface, which lead to a decease of surface band bending. Thus, this provides evidence that the plasma treatment leads to the decrease in an effective Schottky barrier height at the interface of Ti with $n$-type GaN.

Angle resolved analysis was employed to measure the stoichiometry of the GaN at the near-surface region. Varying the angle of detection, $\grave{e}$, yields non-destructive depth information for the GaN samples [15]. At largè̀, the photoelectron signal is mainly contributed from the bulk $\mathrm{GaN}$, while the contribution from the surface region becomes dominant at the smaller $\grave{e}$. The $\mathrm{Ga} / \mathrm{N}$ ratio was calculated by integrating the Ga $3 d$ and $\mathrm{N} 1 s$ photoelectron peaks. The variation of the $\mathrm{Ga} / \mathrm{N}$ ratio with the different surface treatment steps was plotted, as shown in Figure 2. The value of the Ga/N ratio at $\grave{e}=$ $90^{\circ}$ was set to 1.0 for reference. The $\mathrm{Ga} / \mathrm{N}$ ratio changes with $\grave{e}$ in the same trend. At $\grave{e}=30^{\circ}$, the increase in the $\mathrm{Ga} / \mathrm{N}$ ratio is more pronounced after the plasma treatment. This implies that nitrogen atoms preferentially outdiffused during the $\mathrm{Cl}_{2}$ plasma treatment, resulting in the production of $\mathrm{N}$ vacancies at the near surface region.

$\mathrm{The} \mathrm{Ga} / \mathrm{N}$ ratio in the samples treated with $\mathrm{ICP}+\mathrm{HCl}$ is higher than that in the samples treated with ICP, as shown in Figure 2. This might be due to the chemical composition in surface oxides produced during the plasma treatment being different from the that of thermally grown oxides in as-grown sample. During the 
plasma treatment, oxides composed of Ga-rich and Ncontaining $\mathrm{Ga}$ oxides could be produced at the plasmatreated surface. If the subsequent $\mathrm{HCl}$ treatment was effective in selectively removing the $\mathrm{N}$-containing $\mathrm{Ga}$ oxide, this could cause the increase of the $\mathrm{Ga} / \mathrm{N}$ ratio. It was also observed that the $\mathrm{Ga} / \mathrm{N}$ ratio obtained at $60^{\circ}$ is lower than 1.0. This also might be related to changes of chemical composition in the surface oxide with the type of surface treatment. However, further work is needed to clarify this.

\subsection{Mechanism for ohmic contact formation}

The creation of $\mathrm{N}$ vacancies at the plasma-treated surface could be explained through the formation of $\mathrm{Ga}$ oxides during the plasma treatment. In Figure 2(b), the peak intensity of the $\mathrm{O} 1 \mathrm{~s}$ spectra was increased after the $\mathrm{Cl}_{2}$ plasma treatment. This means that $\mathrm{Ga}$ oxides were produced during the treatment. The binding energy of the Ga-O bond is higher than that of Ga-N. Thus, the vaporization of $\mathrm{Ga}$ atoms could be suppressed due to the formation of $\mathrm{Ga}-\mathrm{O}$ bonds, leading to the predominant release of $\mathrm{N}$ atoms. Consequently, the surface of n-type $\mathrm{GaN}$ becomes Ga-rich by the treatment, resulting in the production of $\mathrm{N}$ vacancies at the near surface region.

The dramatic improvement in contact resistivity owing to the pretreatment using $\mathrm{Cl}_{2}$ plasma is explained with the energy band diagram below the interface of $\mathrm{Ti}$ / n-type GaN. The Schottky barrier height of Ti contacts on n-type $\mathrm{GaN}$ is high as about $0.58 \mathrm{eV}$ [16], which is not enough to yield a room temperature ohmic contact [17]. Thus, the contact formed after the pretreatment using $\mathrm{HCl}$ solution results in a rectifying contact, as shown in Figure 3(a). When the surface is treated with the $\mathrm{Cl}_{2}$ plasma, a number of $\mathrm{N}$ vacancies are created at the treated surface. The $\mathrm{N}$ vacancies are located at the shallow level with an energy about $30 \sim 40 \mathrm{meV}$ below the conduction band edge [18]. Thus, Fermi level shifts to the energy level of $\mathrm{N}$ vacancies via the decrease of band bending, as shown in Figure 3(b), leading to the reduction of SBH for transport of electrons. In addition, the $\mathrm{N}$ vacancies produced during the treatment could be autoionized immediately into a hydrogenic configuration due to the atmospheric temperature of about $500 \mathrm{~K}$ in the ICP chamber. The electron concentration near the surface becomes high. Thus, both a reduction of $\mathrm{SBH}$ and an increase of the electron concentration lead to the dramatic decrease in the contact resistivity.

Although Ga oxides exist between the Ti layer and the n-type GaN, one can obtain this low contact resistivity on n-type $\mathrm{GaN}$. In previous work on $\mathrm{GaO}$ on $\mathrm{GaN}$, it was found that only $30-\AA$ of $\mathrm{GaO}$ [19] was formed even after annealing at $800{ }^{\circ} \mathrm{C}$ for $5 \mathrm{~h}$ under dry $\mathrm{O}_{2}$ atmosphere. The oxides produced by the plasma treatment might be much thinner than the thermally grown one, which is enough to transport electrons by thermionic field emission at the interface of $\mathrm{Ti}$ on n-type $\mathrm{GaN}$. However, further work is needed on this.

\section{Conclusions}

A room temperature $\mathrm{Ti} / \mathrm{Al}$ ohmic contact on n-type GaN was demonstrated through the surface treatment using the $\mathrm{Cl}_{2}$ inductively coupled plasma. A specific contact resistance around $\sim 10^{-6} \Omega \mathrm{cm}^{2}$ on n-type GaN was obtained by pretreatment of the surface before deposition of the metals. From the SRPES, it was found that a number of $\mathrm{N}$ vacancies were produced during the plasma treatment. This plays a critical role in reducing contact resistivity dramatically because of self-generation of electrons as well as Fermi level shifting to the higher energy level.

\section{ACKNOWLEDGMENTS}

This work was supported in part by the Korea Institute of Science and Technology Evaluation and Planning (KISTEP) through the NRL projects.

\section{REFERENCES}

[1] Shuji Nakamura, Takashi Mukai, Masayuki Senoh, Appl. Phys. Lett. 64, 1687-1689 (1994).

[2] S. Nakamura, M. Senoh, S. Nagahama, N. Iwasa, T. Yamada, T. Matsushita, Y. Sugimoto, H. Kiyoku, Appl. Phys. Lett. 69, 4056-4058 (1996).

[3] A. Osinsky, S. Gangopadhyay, R. Gaska, B. Williams, J. W. Yang, M. A. Khan, D. Kuksenkov, H. Temkin, Appl. Phys. Lett. 71, 2334-2336 (1997).

[4] M. Asif Khan, J. N. Kuznia, A. R. Bhattarai, D. T. Olson , Appl. Phys. Lett. 62, 1786-1787 (1993).

[5] M. Asif Khan, A. Bhattarai, J. N. Kuznia, D. T. Olson, Appl. Phys. Lett. 63, 1214-1215 (1993).

[6] M. A. Khan, X. Hu, A. Tarakji, G. Simin, J. Yang, R. Gaska, M. S. Shur, Appl. Phys. Lett. 77, 1339-1341 (2000).

[7] M. S. Shur, A. D. Bykhovski, R. Gaska, M. A. Khan, J. W. Yang, Appl. Phys. Lett. 76, 3298-3300 (2000).

[8] M. E. Lin, Z. Ma, F. Y. Huang, Z. F. Fan, L. H. Allen, H. Morkoc , Appl. Phys. Lett. 64, 1003-1005 (1994).

[9] Zhifang Fan, S. Noor Mohammad, Wook Kim, Ozgur Aktas, Andrei E. Botchkarev, Hadis Morkoc, Appl. Phys. Lett. 68, 1672-1674 (1996).

[10] S Ruvimov, Z Liliental-Weber, J Washburn, KJ Duxstad, EE Haller, Z-F Fan, SN Mohammad, W Kim, AE Botchkarev, H Morkoc, Appl. Phys. Lett. 69, 1556-1558 (1996).

[11] J. K. Kim, J. -L. Lee, J. W. Lee, H. E. Shin, Y. J. Park, T. Kim, Appl. Phys. Lett. 73, 2953-2955 (1998).

[12] J. -L. Lee, M. Weber, J. K. Kim, J. W. Lee, Y. J. Park, T. Kim, K. Lynn, Appl. Phys. Lett. 74, 2289-2291 (1999).

[13] A. T. Ping, Q. Chen, J. W. Yang, M. A. Khan, I. Adesida, J. Electron. Mater. 27, 261-265 (1998).

[14] D.J. King, L. Zhang, J.C. Ramer, S.D. Hersee, L.F. Lester, Mater. Res. Soc. Symp. Proc. 468, 421 (1997). 
[15] J. B. Lumsden, in , (American Society for Metals, Metals Park, Ohio, 1986) 568-580.

[16] S. C. Binari, H. B. Dietrich, G. Kelner, L. B. Rowland, K. Doverspike, D. K. Gaskill, Electron. Lett. 30, 909-911 (1994).

[17] D. C. Look, D. C. Reynolds, J. W. Hemsky, J. R. Sizelove, R. L. Jones, R. J. Molnar, Phys. Rev. Lett. 79, 2273 (1997).

[18] P. Boguslawski, E. L. Briggs, J. Bernholc, Phys. Rev. B 51, 17255-17258 (1995).

[19] S. D. Wolter, J. M. DeLuccaa, S. E. Mohney, R. S. Kernb, C. P. Kuo, Thin Sol. Films 371, 153-160 (2000).

\section{FIGURES}

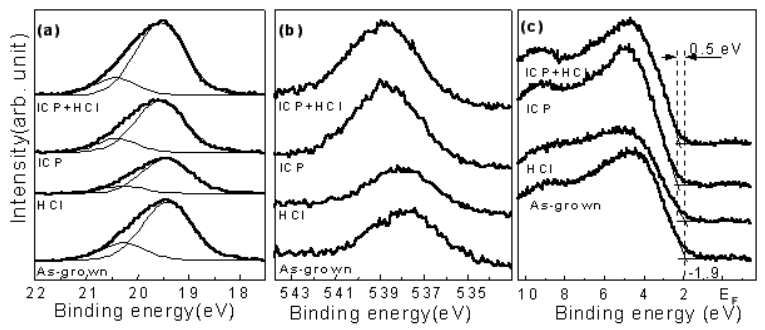

Figure 1. SRPES spectra: (a) Ga $3 d$ photoelectrons, (b) O $1 s$ photoelectrons and (c) valence bands with surface treatments. The position of valence band maximum was determined by the linear extrapolation as shown in (c).

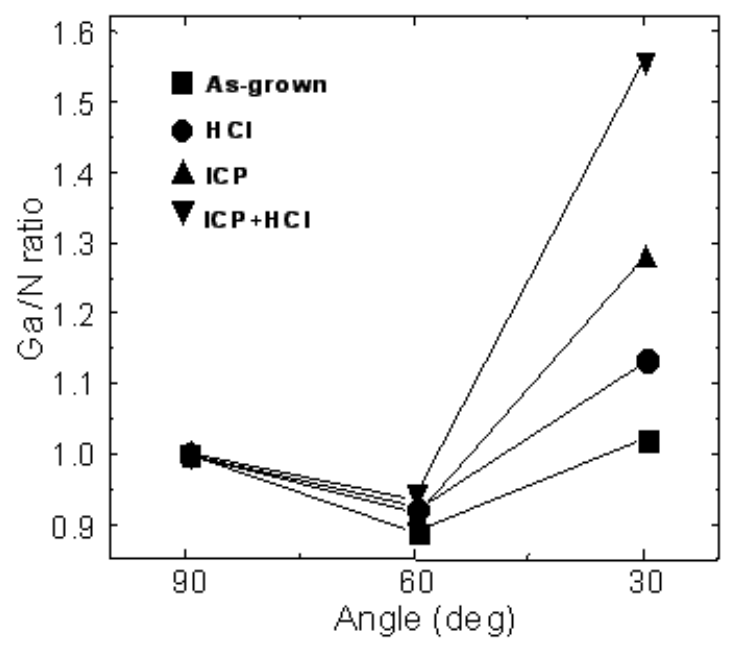

Figure 2. Change of $\mathrm{Ga} / \mathrm{N}$ atomic ratio with the detection angle. The surface normal of sample was defined as $90^{\circ}$.

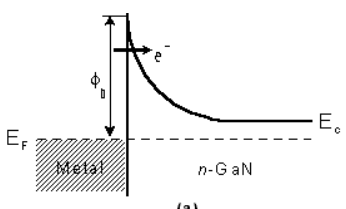

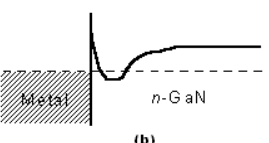

Figure 3. Schematic of energy band diagrams below the interfaces of Ti/n-type GaN: (a) the HCl-treated contact and (b) the plasma-treated contact.

\section{TABLES}

Table I. Resistances as a function of distance of ohmic pads for both HCl-treated and plasma-treated samples.

\begin{tabular}{|c|c|c|}
\hline Distance $(\mu \mathrm{m})$ & HCl-treated $(\Omega)$ & Plasma-treated $(\Omega)$ \\
\hline 3 & $2.14 \times 10^{6}$ & 0.88 \\
\hline 6 & $2.30 \times 10^{6}$ & 1.12 \\
\hline 9 & $2.78 \times 10^{6}$ & 1.28 \\
\hline 12 & $3.11 \times 10^{6}$ & 1.44 \\
\hline 15 & $3.56 \times 10^{6}$ & 1.65 \\
\hline 18 & $3.82 \times 10^{6}$ & 1.88 \\
\hline
\end{tabular}

Table II. FWHMs of the Ga-O bonds with the type of surface treatment

\begin{tabular}{|c|c|c|c|c|}
\hline Sample & As-grown & $\mathrm{HCl}$ & ICP & ICP+HCl \\
\hline FWHM $(\mathrm{eV})$ & 0.97 & 0.96 & 0.83 & 0.77 \\
\hline
\end{tabular}

